\title{
Fluid infiltration pressure for hydrophobic nanochannels
}

\author{
Jingwen Mo, ${ }^{1}$ Long Li,${ }^{1}$ Jianfeng Zhou, ${ }^{1,2}$ Dongyan Xu, ${ }^{3}$ Baoling Huang, ${ }^{1}$ and Zhigang $\mathrm{Li}^{1}{ }^{1, *}$ \\ ${ }^{1}$ Department of Mechanical and Aerospace Engineering, The Hong Kong University of Science and Technology, \\ Clear Water Bay, Kowloon, Hong Kong \\ ${ }^{2}$ College of Mechanical and Power Engineering, Nanjing Tech University, Nanjing 211816, P.R. China \\ ${ }^{3}$ Department of Mechanical and Automation Engineering, The Chinese University of Hong Kong, Shatin, New Territories, Hong Kong
}

(Received 20 January 2015; published 31 March 2015)

\begin{abstract}
We investigate water infiltration pressure for hydrophobic nanochannels through molecular dynamics simulations. It is found that the entrance energy barrier significantly raises the infiltration pressure, which makes the classic Young-Laplace equation invalid for nanochannels. As the channel surface is tuned from superhydrophobic to hydrophobic, the infiltration pressure is greatly reduced mainly due to the decrease of the capillary pressure (Young-Laplace equation) caused by the contact angle change, while the contribution of the entrance energy barrier to the infiltration pressure, which is termed entrance barrier pressure, increases from $25 \%$ to $60 \%$.
\end{abstract}

DOI: 10.1103/PhysRevE.91.033022

PACS number(s): 47.61.-k, 47.55.nb

\section{INTRODUCTION}

Nanofluidics has attracted great interest in the past decades due to its wide applications in science and engineering, including biomolecule manipulation, thermal management of electronic devices, and energy conversion [1-5]. These applications benefit from the high surface-area-to-volume ratio in nanoconfinements, which offers rich opportunities to develop different flow fashions through tuning surface properties [6-9]. However, nanofluidic systems may have inherent problems associated with the small size of nanoconfinements. A notable example is that a high infiltration pressure, $p_{\text {inf }}$, is required to drive fluids into low-surface-energy nanochannels (e.g., hydrophobic channels). According to the classic YoungLaplace equation, the infiltration pressure per unit width for large slit channels is given by [10]

$$
p=-\frac{2 \gamma \cos \theta}{H}
$$

where $\gamma$ and $\theta$ are the surface tension and contact angle of the fluid, and $H$ is the channel height. For hydrophobic channels, where $\theta>90^{\circ}$, as shown in Fig. 1, Eq. (1) indicates that the infiltration pressure is three orders of magnitude higher when the channel size is reduced from micro- to nanoscale. Although Eq. (1) may not be valid for nanochannels, it has been reported that the pressure needed to drive water into carbon nanotubes (CNTs) at room temperature ranges from around 50 to $300 \mathrm{MPa}$, depending on the size of CNTs [11-13]. For nanoporous zeolite with pore size of about 1 $\mathrm{nm}$, the infiltration pressure of water at room temperature is about 80-150 MPa [14,15]. Such high infiltration pressures pose challenges for fabrication and therefore may hinder the applications of nanofluidic systems.

In the past few years, experiments and molecular dynamics (MD) simulations have been conducted to understand fluid infiltration into nanochannels. Both computed and measured infiltration pressures are higher than the predictions of Eq. (1)

*mezli@ust.hk
[11-18]. Other than the work in Refs. [11-15], experiments on water infiltration into nanoscale hydrophobic silica pores show that the infiltration pressures are about 1.5-2 times higher than the Young-Laplace equation [16]. To reduce the discrepancy between experiments and theory, Eq. (1) has been modified by incorporating a disjoining pressure [16] or column resistance [17]. It has been shown that the infiltration pressure depends on the surface properties, temperature, and geometry of nanopores $[11,12,14,15,19-23]$. Molecular dynamics simulations have mainly been used to study how the pore size and temperature affect the fluid infiltration in CNTs, where the surface properties are invariant $[11,12,21,22]$. The general effects of surface properties have been examined by experiments, which have demonstrated that $p_{\text {inf }}$ can be changed through surface treatment; however, the detailed dependence of $p_{\text {inf }}$ on the surface properties or contact angle still remains unknown [19,20]. Our previous work on the flows in nanochannels reveals that the molecular interactions between fluid molecules and wall atoms play a critical role and make the classic Navier-Stokes equation invalid in certain conditions $[6,24,25]$. Given the fluid, such molecular interactions are mainly dependent on the surface energy, which is also expected to be important in affecting the fluid infiltration into nanochannels because the fluid contact angle is largely determined by the surface energy. In addition, surface properties also affect the potential energy distribution at the channel entrance, which is also related to the infiltration pressure. Unfortunately, how the molecular interactions and surface properties affect the fluid infiltration pressure for hydrophobic nanochannels is unclear.

In this work, we study water infiltration into nanochannels and investigate the infiltration pressure $p_{\text {inf }}$ through MD simulations. It is found that the entrance energy barrier plays an important role at the nanoscale and the Young-Laplace equation [Eq. (1)] underestimates the infiltration pressure. As the surface energy is varied and the surface is changed from superhydrophobic to hydrophobic, the pressure due to the entrance energy barrier, which is referred to as the entrance barrier pressure, drops gently; however, $p_{\text {inf }}$ is greatly reduced by a factor of 7 in $3 \mathrm{~nm}$ channels due to the decrease of contact angle. 


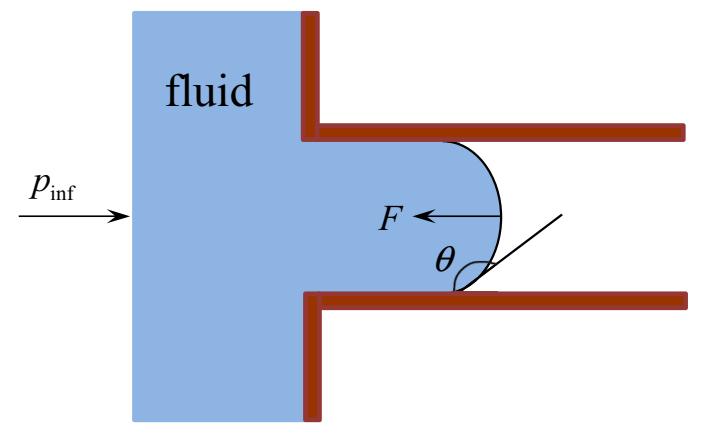

FIG. 1. (Color online) Schematic of fluid infiltration into a hydrophobic channel.

\section{MOLECULAR DYNAMICS SIMULATIOIN}

The MD simulation system consists of a reservoir and a nanochannel, as illustrated in Fig. 2. The size of the system is 6.1 and $4.9 \mathrm{~nm}$ in the $y$ and $z$ directions, respectively. Initially, the reservoir is $9.8 \mathrm{~nm}$ long in the $x$ direction. It is filled with a certain number of water molecules and constrained by two rigid walls (Fig. 2). The nanochannel is formed by two parallel walls, which are constructed by truncating a rectangular portion from a face-centered cubic (fcc) structure with a lattice constant equal to $4.086 \AA$. Each wall contains four atomic layers. The outmost layers are fixed to maintain a stable system, while the other interior layers are free to vibrate to account for the flexibility of the walls and control the temperature of the system. The channel height and length are 3 and $9.8 \mathrm{~nm}$. Periodic boundary conditions (PBCs) are employed in the $y$ and $z$ directions for the water in the reservoir and in the $z$ direction for the channel.

The extended simple point charge potential (SPC/E) is used to model water molecules [26], which assumes a rigid tetrahedral shape for water molecules with $\mathrm{H}-\mathrm{O}-\mathrm{H}$ angle of $109.47^{\circ}$ and a fixed O-H distance of $1 \AA$. Initially the oxygen atoms are placed on fcc lattice sites. The positions of hydrogen atoms are determined based on the oxygen positions with rotations calculated using Hamilton's quaternions [27]. Each oxygen and hydrogen atom carry a point charge $q$ equal to -0.8476 e and $0.4238 \mathrm{e}$, respectively. The potential for any two interacting water molecules, $a$ and $b$, is given by

$$
U_{a b}=\sum_{i \in a} \sum_{j \in b} \frac{k_{c} q_{i} q_{j}}{r_{i j}}+\left[\left(\frac{A}{r_{o o}}\right)^{12}-\left(\frac{B}{r_{o o}}\right)^{6}\right], i, j=1,2,3,
$$

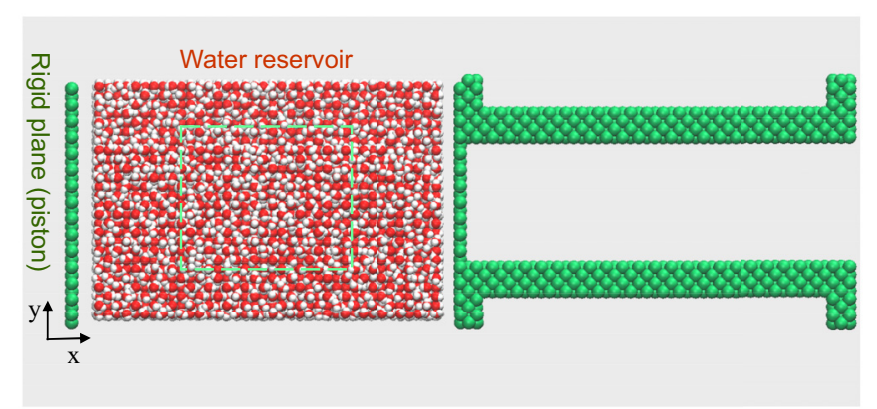

FIG. 2. (Color online) The initial state of the molecular dynamics simulation system. where $k_{c}=1389.5064 \mathrm{~kJ} / \mathrm{mol} \cdot \AA$ is the electrostatic constant, $A=3.428(\mathrm{~kJ} / \mathrm{mol})^{1 / 12} \cdot \AA, B=3.7122(\mathrm{~kJ} / \mathrm{mol})^{1 / 6} \cdot \AA, r_{o o}$ is the separation between the oxygen atoms of the two interacting water molecules, and the subscripts $i$ and $j$ represent the $i$ th and $j$ th atom ( $\mathrm{H}$ or $\mathrm{O}$ ) of water molecules $a$ and $b$, respectively. For the conservation of the total energy, the electrostatic potential is multiplied by a termination function (MEI4) [28] to shift the electrostatic potential and force to be zero at the cutoff distance of $13 \AA$. This scheme is found to produce similar structural and dynamic properties of bulk water to those computed by Ewald summation [29-31]. The interactions among wall atoms are modeled by the tight-binding potential, which has been well accepted for transition metals, and the parameters for Ag are used [32,33]. The water-surface interaction is described by the Lennard-Jones $(\mathrm{LJ})$ potential $U(r)=4 \varepsilon_{\mathrm{fw}}\left[\left(\sigma_{\mathrm{fw}} / r\right)^{12}-\left(\sigma_{\mathrm{fw}} / r\right)^{6}\right]$ between oxygen and wall atoms, where $\varepsilon_{\mathrm{fw}}$ and $\sigma_{\mathrm{fw}}$ are the fluid-wall binding energy and collision diameter. $\sigma_{\mathrm{fw}}=2.85 \AA$ is employed based on the Lorentz-Berthelot mixing rule using the self-interaction LJ parameters for oxygen and Ag given in Refs. [34,35], while $\varepsilon_{\mathrm{fw}}$ is varied to change the water-surface interaction strength for considering the surface effects.

The equations of motion are integrated using Beeman's algorithm [36] with a time step equal to $1 \mathrm{fs}$. The $\mathrm{O}-\mathrm{H}$ bond distance and the H-O-H bond angle of a water molecule are maintained by the constraint dynamics algorithm [37]. The SPC/E model is tested by calculating physical properties of water in a cube with PBCs in all the directions. The MD result for the self-diffusion coefficient of water at room temperature, $D_{\mathrm{H}_{2} \mathrm{O}}=2.88 \times 10^{-9} \mathrm{~m}^{2} / \mathrm{s}$, is consistent with experimental data $\left(2.4 \times 10^{-9} \mathrm{~m}^{2} / \mathrm{s}\right)$ [38]. At $300 \mathrm{~K}$ and $0.1 \mathrm{MPa}$, the water density and potential energy are calculated as $992 \mathrm{~kg} / \mathrm{m}^{3}$ and $-41.7 \mathrm{~kJ} / \mathrm{mol}$, which also agree well with experiments $\left(996.5 \mathrm{~kg} / \mathrm{m}^{3}\right.$ and $\left.-41.7 \mathrm{~kJ} / \mathrm{mol}\right)[39,40]$.

The temperature of the system is maintained at $300 \mathrm{~K}$ by the Berendsen thermostat [37], which is as accurate as the Nosé-Hoover thermostat and does not affect the numerical results because the number of molecules in the simulation system is sufficiently large [7]. Here 9708 water molecules are included in the reservoir to make the water density close to that of bulk water at $1 \mathrm{~atm}$. After 20 ps relaxation, the right wall of the reservoir (Fig. 2) is removed, and the system is relaxed for another $30 \mathrm{ps}$ before the left wall is moved in the positive $x$ direction in a stepwise and quasistatic manner [21]. The left wall is moved one step of $0.3 \AA$ forward every 8 ps, such that the system has sufficiently long time to reach a new equilibrium state. By sampling a large number of water molecules in a cuboid in the reservoir, as indicated by the dashed lines in Fig. 2, the water pressure is calculated based on the virial theorem modified for the case without PBCs [37,41],

$$
\begin{aligned}
p= & \frac{1}{3 \forall}\left[\frac{1}{M} \sum_{\alpha=1}^{N}\left(\sum_{i} m_{i \alpha} v_{i \alpha}\right)^{2}\right. \\
& \left.+\sum_{\substack{\alpha, \beta=1 \\
(\alpha<\beta)}}^{N} r_{\alpha \beta} F_{\alpha \beta}+\sum_{\alpha=1}^{N} \sum_{\gamma} r_{\alpha \gamma} F_{\alpha \gamma}\right],
\end{aligned}
$$




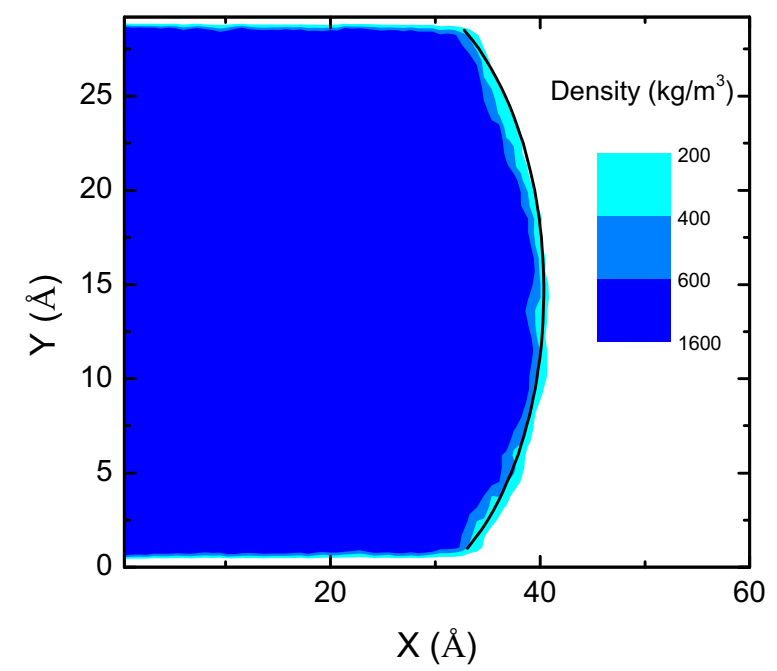

FIG. 3. (Color online) Density contour of water in the nanochannel for $\varepsilon_{\mathrm{fw}}=50 \mathrm{~K}$. The contact angle of water is determined by a series of isopycnic lines. The solid line is the isopycnic line for density equal to $300 \mathrm{~kg} / \mathrm{m}^{3}$.

where $\forall$ is the sampling volume, $M$ is the mass of a water molecule, $N$ is the total number of water molecules in the cuboid, subscript $i$ denotes the $i$ th atom (either $\mathrm{H}$ or $\mathrm{O}$ ) of water molecule $\alpha$, and $r$ and $F$ are the mass center distance and total force between water molecules $\alpha$ and $\beta$ or $\gamma(\gamma$ represents the neighboring molecules outside of the sampling volume). The pressures calculated using Eq. (3) are in good agreement with those obtained by computing the total force acting on the left wall of the reservoir.

To obtain the contact angle $\theta$, water molecules are initially arranged to fill the reservoir and half of the channel. The left wall of the reservoir is kept stationary throughout the simulation. The channel is divided into many $0.7 \times 0.7 \times$ $12.3 \AA$ cuboids. At equilibrium, water density of each cuboid is averaged over $50 \mathrm{ps}$, and the meniscus of water is fitted by isopycnic lines of density ranging from 200 to $600 \mathrm{~kg} / \mathrm{m}^{3}$ with an increment of $100 \mathrm{~kg} / \mathrm{m}^{3}$ [42]. The contact angles for the isopycnic lines are obtained and the average value is considered as the contact angle. Figure 3 shows the density distribution of water in the channel and the isopycnic line of density equal to $300 \mathrm{~kg} / \mathrm{m}^{3}$.

\section{RESULTS AND DISCUSSION}

The infiltration pressure $p_{\text {inf }}$ is determined by monitoring the pressure $p$ of the reservoir and the number of water molecules infiltrated into the channel $N_{\text {inf }}$ at room temperature ( $T=300 \mathrm{~K}$ ). A typical plot of $p$ versus $N_{\text {inf }}$ for a hydrophobic channel is depicted in Fig. 4, where the water-surface binding energy $\varepsilon_{\mathrm{fw}}=30 \mathrm{~K}$ (the contact angle $\theta=140^{\circ}$, as shown later). It is seen that water molecules do not infiltrate into the channel until $p$ reaches about $10 \mathrm{MPa}$. After that, $N_{\text {inf }}$ increases linearly with $p$ till $p$ reaches around $45 \mathrm{MPa}$, where water molecules are continuously driven into the channel while $p$ remains almost constant. This constant pressure is referred to as the infiltration pressure $p_{\text {inf }}$.

In Fig. 4 , it is clear that $p_{\text {inf }}$ is contributed by two parts. One is the first critical pressure $(\sim 10 \mathrm{MPa})$, below which

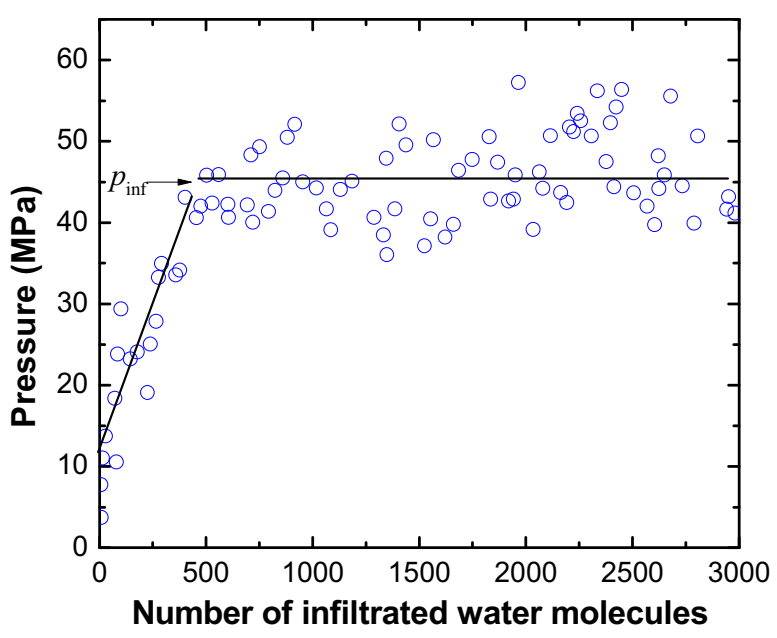

FIG. 4. (Color online) Number of water molecules infiltrated into the nanochannel versus the pressure of the reservoir for $\varepsilon_{\mathrm{fw}}=30 \mathrm{~K}$.

water infiltration does not occur. This pressure is caused by the potential energy barrier at the channel entrance due to the water-wall molecular interactions. At room temperature, the average kinetic and potential energies of water molecules are around 0.1 and $-0.4 \mathrm{eV}$, respectively. This means that water molecules will have difficulty in diffusing to regions with potential higher than $-0.3 \mathrm{eV}$ (total energy of water molecules) without the help of external forces. Figure 5(a) depicts the potential distribution in an $x y$ plane near the bottom wall at the channel entrance for the case in Fig. 4. It is seen that the potential is positive in most of the regions, which sets an energy barrier and water molecules have to overcome it to infiltrate into the channel. This critical pressure is designated as the entrance barrier pressure, $p_{\Delta E}$, which can be obtained by fitting the linear part of the pressure (Fig. 4) and extrapolating it to the $y$ axis. This gives that $p_{\Delta E}=11.5 \mathrm{MPa}$ for the case in Fig. 4 , which is about $25 \%$ of the infiltration pressure $p_{\text {inf }}$. As the pressure is higher than $p_{\Delta E}$, water molecules start to infiltrate into the channel and the water meniscus gradually forms, which corresponds to the linear increase part in Fig. 4. After sufficient water molecules are driven into the channel and the water meniscus is completely formed, the pressure remains nearly a constant. This second part is caused by the surface tension, i.e. the capillary pressure, as predicted by Eq. (1). Therefore, at the nanoscale, the entrance barrier pressure $p_{\Delta E}$ and the capillary pressure contribute to the infiltration pressure $p_{\text {inf }}$.

Since the entrance energy barrier and capillary pressure strongly depend on the channel surface energy, the infiltration pressure $p_{\text {inf }}$ is obtained by tuning the surface hydrophobicity, which is realized by changing the $\varepsilon_{\mathrm{fw}}$ value. Figure 6 shows $p_{\text {inf }}$ and contact angle $\theta$ as a function of $\varepsilon_{\mathrm{fw}}$. It is seen that $p_{\text {inf }}$ decreases monotonously from 48.5 to $7 \mathrm{MPa}$ when $\varepsilon_{\mathrm{fw}}$ is increased from 10 to $130 \mathrm{~K}$, for which the contact angle $\theta$ decreases almost linearly from about $150^{\circ}$ to $90^{\circ}$. The entrance barrier pressure $p_{\Delta E}$ is shown in Fig. 7 as $\varepsilon_{\mathrm{fw}}$ is varied. It is found that $p_{\Delta E}$ decreases gently with increasing $\varepsilon_{\mathrm{fw}}$. This is because the entrance energy barrier drops as $\varepsilon_{\mathrm{fw}}$ is raised, as illustrated in Figs. 5(a) and 5(b) for $\varepsilon_{\mathrm{fw}}=30$ and $130 \mathrm{~K}$, respectively. It is also seen that the contribution of $p_{\Delta E}$ to 

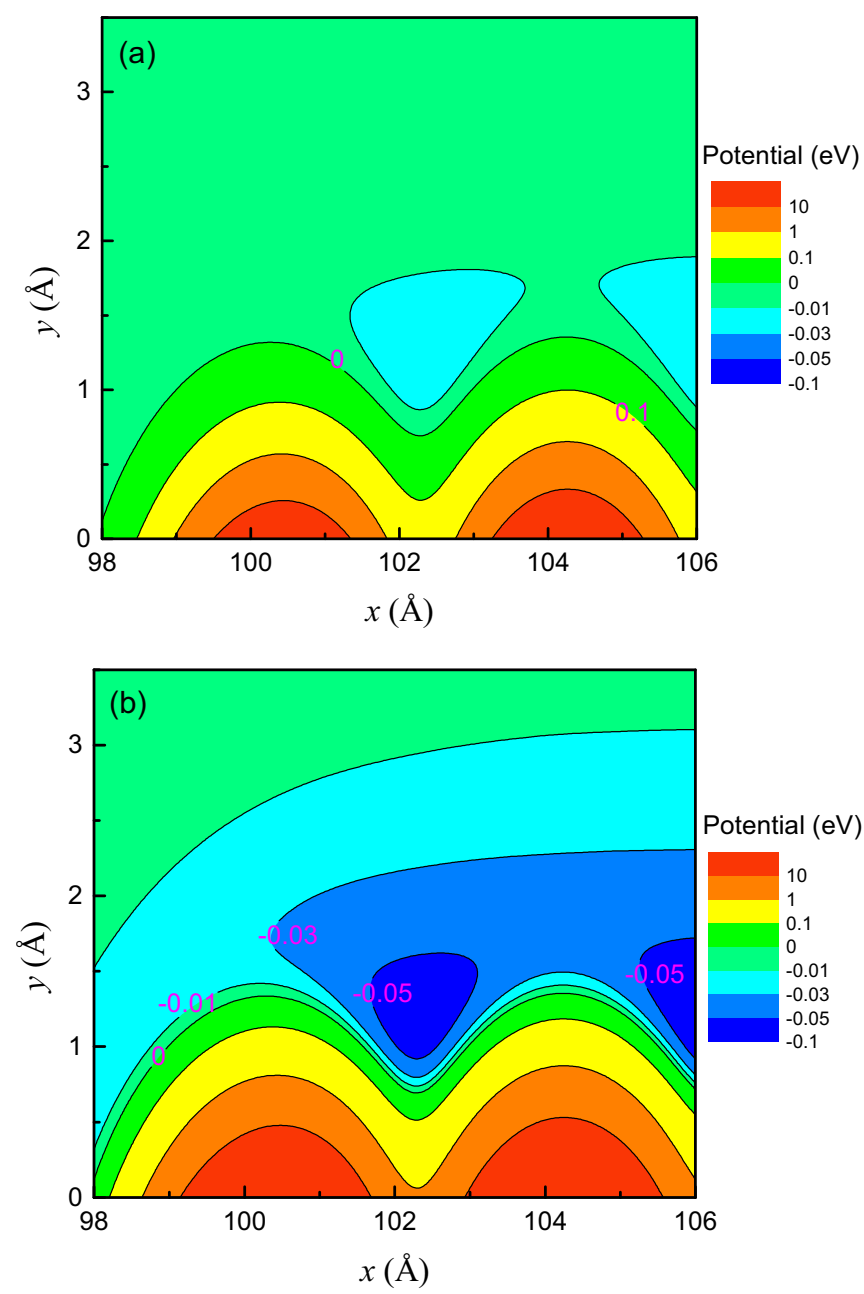

FIG. 5. (Color online) Potential distribution in an $x y$ plane near the bottom wall at the channel entrance (the channel starts at $x=$ $98 \AA$ ). (a) $\varepsilon_{\mathrm{fw}}=30 \mathrm{~K}$ and (b) $\varepsilon_{\mathrm{fw}}=130 \mathrm{~K}$.

the infiltration pressure $p_{\text {inf }}$ ranges from $25 \%$ to $60 \%$ as the contact angle is varied, which is significant.

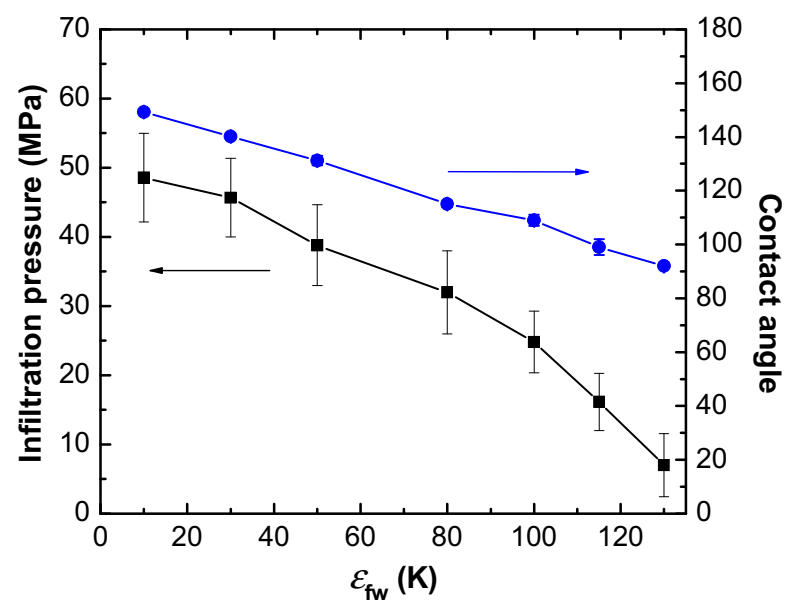

FIG. 6. (Color online) Infiltration pressure and contact angle as a function of water-surface binding energy $\varepsilon_{\mathrm{fw}}$.

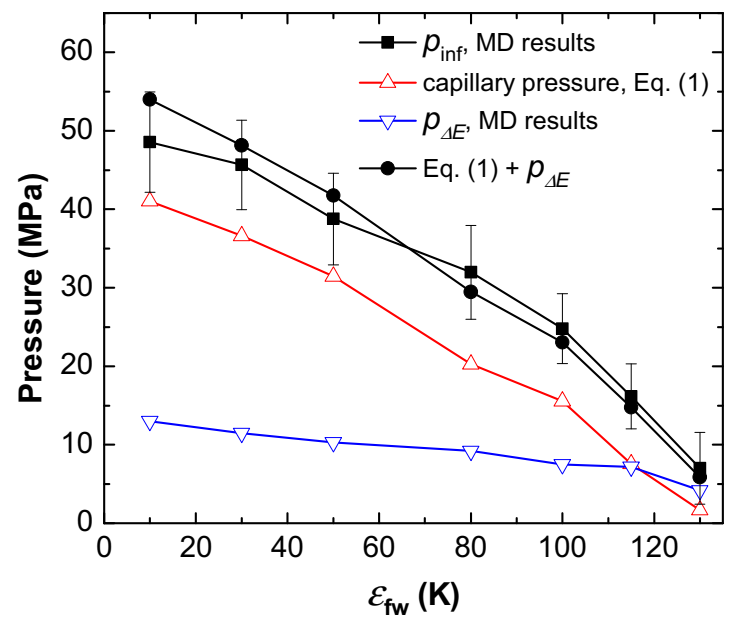

FIG. 7. (Color online) Entrance barrier pressure and capillary pressure as a function of $\varepsilon_{\mathrm{fw}}$.

The contribution of the capillary pressure can be theoretically calculated using Eq. (1). Since water surface tension $\gamma$ has been shown independent of surface energy [43], $\gamma=$ $71.6 \mathrm{mN} / \mathrm{m}[44,45]$ is used for the water surface tension. The capillary pressures predicted by Eq. (1) based on the contact angles in Fig. 6 are shown in Fig. 7, which, as expected, are smaller than $p_{\text {inf }}$. The capillary pressure decrease with increasing $\varepsilon_{\mathrm{fw}}$ in a similar manner to $p_{\text {inf }}$. If the entrance barrier pressure $p_{\Delta E}$ is considered, the total pressure, i.e. the prediction of Eq. (1) and $p_{\Delta E}$, is in good agreement with $p_{\text {inf }}$ computed directly from MD simulations, as shown in Fig. 7. It is worth mentioning that the surface energy is definitely the most important factor in affecting the infiltration pressure as it controls the entrance energy barrier and contact angle. The channel structure at the entrance, channel size, and temperature may also play essential roles. All these parameters determine the critical channel size, where the Young-Laplace equation breaks down. This will be investigated in the future work.

\section{CONCLUSION}

In summary, we have investigated water infiltration into hydrophobic nanochannels at room temperature. In addition to the capillary pressure, the entrance energy barrier also contributes significantly, up to $60 \%$, to the infiltration pressure, depending on the contact angle. As the channel is tuned from superhydrophobic to hydrophobic, the infiltration pressure is greatly reduced, by a factor of seven. Such reduction is mainly caused by the capillary pressure due to the contact angle change.

\section{ACKNOWLEDGMENTS}

This work was supported by the Research Grants Council of the Hong Kong Special Administrative Region under Grant Nos. 615312 and 16205714 . J.M. was partially supported by a Postgraduate Scholarship through the Energy Program at HKUST. 
[1] M. Napoli, J. C. T. Eijkel, and S. Pennathur, Lab Chip 10, 957 (2010).

[2] P. Abgrall and N. T. Nguyen, Anal. Chem. 80, 2326 (2008).

[3] C. Liu and Z. Li, Phys. Rev. Lett. 105, 174501 (2010).

[4] W. H. Duan and Q. Wang, ACS Nano 4, 2338 (2010).

[5] F. H. J. van der Heyden, D. Stein, and C. Dekker, Phys. Rev. Lett. 95, 116104 (2005).

[6] C. Liu and Z. Li, Phys. Rev. E 80, 036302 (2009).

[7] C. Liu and Z. Li, J. Chem. Phys. 132, 024507 (2010).

[8] S. K. Kannam, B. D. Todd, J. S. Hansen, and P. J. Daivis, J. Chem. Phys. 135, 144701 (2011).

[9] L. Li, J. Mo, and Z. Li, Phys. Rev. E 90, 033003 (2014).

[10] R. J. Stokes and D. F. Evans, Fundamentals of Interfacial Engineering (Wiley-VCH, New York, 1997).

[11] B. Xu, Y. Qiao, T. Park, M. Tak, Q. Zhou, and X. Chen, Energ. Environ. Sci. 4, 3632 (2011).

[12] J. Zhao, L. Liu, P. J. Culligan, and X. Chen, Phys. Rev. E 80, 061206 (2009).

[13] L. Liu, Y. Qiao, and X. Chen, Appl. Phys. Lett. 92, 101927 (2008).

[14] Y. Qiao, V. K. Punyamurtula, A. Han, X. Kong, and F. B. Surani, Appl. Phys. Lett. 89, 251905 (2006).

[15] Y. Qiao and A. Han, Phil. Mag. Lett. 87, 25 (2007).

[16] R. Helmy, Y. Kazakevich, C. Ni, and Y. Fadeev, J. Am. Chem. Soc. 127, 12446 (2005).

[17] Y. Qiao, L. Liu, and X. Chen, Nano Lett. 9, 984 (2009).

[18] B. V. Derjaguin, N. V. Churaev, and V. M. Muller, Surface Forces (Consultant Bureau, New York, 1987).

[19] A. Han and Y. Qiao, Chem. Lett. 36, 882 (2007).

[20] A. Han and Y. Qiao, Chem. Phys. Lett. 454, 294 (2008).

[21] L. Liu, J. Zhao, P. J. Culligan, Y. Qiao, and X. Chen, Langmuir 25, 11862 (2009).

[22] L. Liu, J. Zhao, C. Y. Yin, P. J. Culligan, and X. Chen, Phys. Chem. Chem. Phys. 11, 6520 (2009).

[23] L. Coiffard and V. Eroshenko, J. Colloid Interf. Sci. 300, 304 (2006).
[24] C. Liu and Z. Li, AIP Adv. 1, 032108 (2011).

[25] C. Liu, Y. Lv, and Z. Li, J. Chem. Phys. 136, 114506 (2012).

[26] H. J. C. Berendsen, J. R. Grigera, and T. P. Straatsma, J. Phys. Chem. 91, 6269 (1987).

[27] H. Goldstein, Classical Mechanics, 2nd ed. (Addison-Wesley, New York, 1980).

[28] C. L. Brooks, B. M. Pettitt, and M. Karplus, J. Chem. Phys. 83, 5897 (1985).

[29] M. Prevost, D. Van Belle, G. Lippens, and S. Wodak, Mol. Phys. 71, 587 (1990).

[30] P. J. Steinbach and B. R. Brooks, J. Comput. Chem. 15, 667 (1994).

[31] P. Mark and L. Nilsson, J. Phys. Chem. A 105, 9954 (2001).

[32] F. Cleri and V. Rosato, Phys. Rev. B 48, 22 (1993).

[33] Z. Li and H. Wang, Phys. Rev. Lett. 95, 014502 (2005).

[34] S. Chowdhuri and A. Chandra, J. Chem. Phys. 115, 3732 (2001).

[35] Z. Li, Phys. Rev. E 79, 026312 (2009).

[36] Z. Li, Phys. Rev. E 80, 061204 (2009).

[37] M. Allen and D. Tildesley, Computer Simulation of Liquids (Oxford University Press, New York, 1987).

[38] K. Krynicki, C. D. Green, and D. W. Sawyer, Faraday Disc. Chem. Soc. 66, 199 (1978).

[39] E. Schmidt, Properties of Water and Steam in SI-Units (Springer, Berlin, 1969).

[40] J. P. M. Postma, Ph.D. thesis, University of Groningen, the Netherlands, 1985.

[41] H. J. C. Berendsen, J. P. M. Postma, W. F. van Gunsteren, A. DiNola, and J. R. Haak, J. Chem. Phys. 81, 3684 (1984).

[42] M. J. P. Nijmeijer, C. Bruin, A. F. Bakker, and J. M. J. Van Leeuwen, Physica A 160, 166 (1989).

[43] M. J. P. Nijmeijer, C. Bruin, A. F. Bakker, and J. M. J. van Leeuwen, Phys. Rev. A 42, 6052 (1990).

[44] F. Chen and P. E. Smith, J. Chem. Phys. 126, 221101 (2007).

[45] W. R. Haynes, Handbook of Chemistry and Physics, 95th ed. (CRC Press, Boca Raton, FL, 2014). 